\title{
Low-Reynolds-number fountain behaviour
}

\author{
N. WILLIAMSON ${ }^{1}$, N. SRINARA YA A A ${ }^{1}$, \\ S. W. ARMFIELD ${ }^{1}$, G. D. McBA IN ${ }^{1}$ AND W. L I N ${ }^{2}$ \\ ${ }^{1}$ School of Aerospace, Mechanical and Mechatronic Engineering, The University of Sydney, \\ NSW, 2006 Australia \\ ${ }^{2}$ School of Engineering, James Cook University, Townsville, Queensland, 4811 Australia
}

(Received 11 February 2008 and in revised form 20 April 2008)

Experimental evidence for previously unreported fountain behaviour is presented. It has been found that the first unstable mode of a three-dimensional round fountain is a laminar flapping motion that can grow to a circling or multimodal flapping motion. With increasing Froude and Reynolds numbers, fountain behaviour becomes more disorderly, exhibiting a laminar bobbing motion. The transition between steady behaviour, the initial flapping modes and the laminar bobbing flow can be approximately described by a function $\mathrm{Fr} R e^{2 / 3}=C$. The transition to turbulence occurs at $R e>120$, independent of Froude number, and the flow appears to be fully turbulent at $R e \approx 2000$. For $F r>10$ and $R e \lesssim 120$, sinuous shear-driven instabilities have been observed in the rising fluid column. For $R e \gtrsim 120$ these instabilities cause the fountain to intermittently breakdown into turbulent jet-like flow. For $F r \lesssim 10$ buoyancy forces begin to dominate the flow and pulsing behaviour is observed. A regime map of the fountain behaviour for $0.7 \lesssim F r \lesssim 100$ and $15 \lesssim R e \lesssim 1900$ is presented and the underlying mechanisms for the observed behaviour are proposed. Movies are available with the online version of the paper.

\section{Introduction}

Fountains (negatively buoyant jets) occur when a fluid is injected into another fluid of differing density where the buoyancy force opposes the momentum of the flow. The injected fluid penetrates a distance into the environment before stagnating and falling back around itself. The characteristics of the fountain flow, such as the penetration height and the amount of mixing, depend on the Froude number and Reynolds number, which for round fountains are defined as

$$
F r=\frac{U_{0}}{\sqrt{R_{0} \sigma}}
$$

and

$$
R e=\frac{U_{0} R_{0}}{v_{0}},
$$

respectively. $R_{0}$ is the radius of the source and $\sigma$ is the reduced gravity between the fountain source and the ambient fluid and is defined as $\sigma=g\left(\rho_{0}-\rho_{f}\right) / \rho_{f}$. The subscript 0 indicates a quantity at the fountain source and $f$ indicates a property of the ambient fluid. The characteristic velocity $U_{0}$ is defined here as $U_{0}=Q_{0} / \pi R_{0}^{2}$, where $Q_{0}$ is the volume flow rate at the fountain source; $v_{0}$ is the kinematic viscosity of the fluid at the fountain source. 
Fountain flow is generally categorized as weak for $\mathrm{Fr} \lesssim 1$ and forced for $\mathrm{Fr} \gtrsim 3$ (Kaye \& Hunt 2006). Weak fountains are generally steady (Lin \& Armfield 2000a) and symmetric, whereas forced fountains penetrate a greater distance into the ambient fluid and are unsteady with stronger mixing and entrainment of ambient fluid (Turner 1966; Friedman et al. 2007).

There have been numerous studies investigating fountain behaviour in a variety of configurations including round fountains (Turner 1966), planar fountains (Zhang \& Baddour 1997; Lin \& Armfield 2000b; Srinarayana et al. 2007), fountains impinging on a solid surface and fountains penetrating an interface (Friedman 2006). Most early studies have focused on forced turbulent round fountains which arise in numerous industrial and environmental flows as diverse as flow in magma chambers (Campbell \& Turner 1989), air-conditioning and heating in large buildings (Baines, Turner \& Campbell 1990, among many others), flow from chimneys, effluent dispersion from pipes in the ocean, volcanic eruptions (Kaminski, Tait \& Carazzo 2005) and some atmospheric flows (Turner 1966). Significant contributions have been made by Turner (1966), Abraham (1967), Mizushina et al. (1982), Baines et al. (1990) and Bloomfield \& Kerr (2000). The consensus in these works, on both dimensional (Turner 1966), analytical (Abraham 1967) and experimental grounds (Turner 1966; Mizushina et al. 1982; Baines et al. 1990), is that for forced turbulent round fountains the maximum penetration depth $Z_{m}$ scales as $Z_{m} / R_{0}=C F r$, where $C$ is a constant of proportionality, and is Reynolds-number-independent. Turner (1966) performed the first experimental work on turbulent round fountains using salt-water solution injected upwards into a fresh-water tank and found $C=2.46$ over the range $2 \lesssim F r \lesssim 30$. Other experimental studies followed which confirmed the linear relationship, albeit with some small variation in the magnitude of the coefficient. Mizushina et al. (1982) obtained $C=2.35$ for $5<F r<260$ and $1130<R e<2710$; Campbell \& Turner (1989) found $C=2.46$; Baines et al. (1990) found $C=2.46$ for $10<F r<300$ and $R e \sim 2000$; and Pantzlaff \& Lueptow (1999) found $C=2.1$ for $15 \leqslant F r \leqslant 78$ and $1250 \leqslant R e \leqslant 7500$. The description of the flows provided in these experimental studies is that the flow becomes turbulent close to the source and the flow is generally quite stable, with only small oscillations in the fountain height after an initial transient period has passed.

More recently attention has been given to turbulent weak fountains (Zhang \& Baddour 1998; Lin \& Armfield 2000a,c; Kaye \& Hunt 2006; Friedman et al. 2007), weak and forced laminar fountains (Lin \& Armfield 2003; Philippe et al. 2005) and transitional fountains (Lin \& Armfield 2004). Across these studies there is much less agreement in the form of the scaling for $Z_{m}$ than for forced turbulent fountains. Many authors have suggested sub-ranges over which scalings are valid, suggesting that the dominant mechanisms governing the flow are dependent on both the Reynolds and Froude numbers. These scalings have been summarized in table 1 . It has been reported that the transition from laminar to turbulent flow occurs at $60<R e<200$ (Philippe et al. 2005) and in most studies fountain flow is considered fully turbulent with $R e \gtrsim 1100-2000$ (Pantzlaff \& Lueptow 1999; Mizushina et al. 1982; Baines et al. 1990).

Lin \& Armfield (2000a) numerically simulated fountains with $0.2 \leqslant F r \leqslant 1.0$ and $R e=200$. At these very low Froude numbers the fountain flow is very weak and penetrates only a very small distance before reversing and flowing radially out from the source. The intrusion layer formed is thick relative to the fountain height and is an important factor in the flow. In this study the authors found that a linear Froude number scaling for the penetration height, i.e. $Z_{m} / R_{0} \sim F r$, is still valid. The authors also simulated fountain flow for $1.0 \leqslant F r \leqslant 2.0$ and found the linear scaling to be 
Turbulent fountains

Turner (1966)

$$
Z_{m} / R_{0} \sim F r, \quad F r>2.0
$$

Transitional/turbulent fountains

\begin{tabular}{|c|c|c|c|}
\hline Zhang \& Baddour (1998) & $Z_{m} / R_{0} \sim$ & $\left\{\begin{array}{l}F r, \\
F r^{1.3},\end{array}\right.$ & $\begin{array}{l}F r \gtrsim 7.0 \\
F r \lesssim 7.0\end{array}$ \\
\hline Lin \& Armfield (2004) & $Z_{m} / R_{0} \sim$ & $\begin{array}{l}F r \operatorname{Re}^{1 / 4}, \\
\mathrm{Fr}\end{array}$ & $\begin{array}{l}1 \leqslant F r \leqslant 8,200 \leqslant R e \leqslant 800 \\
F r \gtrsim 3.0\end{array}$ \\
\hline aye \& Hunt (2006) & $Z_{m} / R_{0} \sim$ & $\left\{\begin{array}{l}\mathrm{Fr}^{2} \\
\mathrm{Fr}^{2 / 3}\end{array}\right.$ & $\begin{array}{l}1.0 \lesssim F r \lesssim 3.0 \\
F r \lesssim 1.0\end{array}$ \\
\hline
\end{tabular}

Laminar/transitional fountains

Lin \& Armfield (2000c)

Lin \& Armfield (2000a)

Lin \& Armfield (2003)

Philippe et al. (2005)

$$
\begin{aligned}
& Z_{m} / R_{0} \sim(\mathrm{Fr} / \mathrm{Re})^{2 / 3}, \\
& Z_{m} / R_{0} \sim \mathrm{Fr}, \\
& Z_{m} / R_{0} \sim \mathrm{Fr} / \mathrm{Re}^{1 / 2}, \\
& Z_{m} / R_{0} \sim \mathrm{Fr} \mathrm{Re}^{1 / 2},
\end{aligned}
$$

$0.0025 \leqslant F r \leqslant 0.2,5 \leqslant R e \leqslant 800$

$0.2 \leqslant F r \leqslant 1.0, R e=200$

$5 \leqslant R e \leqslant 200, F r=1.0$

$1 \lesssim F r \lesssim 200,0<R e<80$

TABLE 1. Proposed scaling of fountain mean penetration height grouped by Reynolds number. Here the flow is classified as turbulent for $R e>2000$, transitional for $100<R e<2000$ and laminar for $R e<100$.

invalid. Kaye \& Hunt (2006) later re-plotted these results and found them to be well fitted by $Z_{m} / R_{0} \sim F r^{2 / 3}$ for $F r<1$ and $Z_{m} / R_{0} \sim F r^{2}$ for $F r>1$. In a subsequent study Lin \& Armfield (2003) examined the effects of viscosity on the flow. By reducing the radial momentum equation down to a balance between the radial pressure gradient in the intrusion outflow and viscous dissipation therein, the authors argue that $Z_{m} / R_{0} \sim F r / R e^{1 / 2}$. The authors conducted numerical simulations for $5 \leqslant R e \leqslant 800$ at $F r=1.0$ and found that below $R e=200$ this scaling was well supported by the numerical results. In Lin \& Armfield $(2000 c)$, the authors examined very weak fountains using numerical simulations with $0.0025 \leqslant F r \leqslant 0.2$ and $R e=200$ and found $Z_{m} / R_{0} \sim \mathrm{Fr}^{2 / 3}$. The authors argued that in very-low-Froude-number fountains inertial effects are small, so dimensional consistency requires $Z_{m} / R_{0} \sim\left(v^{2} / \sigma\right)^{1 / 3}$. The authors performed simulations for $5 \leqslant R e \leqslant 800$, with $F r=0.05$ and found $Z_{m} / R_{0}=0.1615+$ $0.3803 R e^{-2 / 3}$. This supports their scaling at very low Reynolds numbers, but above $R e=50$ the Reynolds-number effect becomes quite weak and the constant term dominates the correlation.

Philippe et al. (2005) experimentally examined laminar forced fountains for $1 \lesssim F r \lesssim 200$ and $0<R e<80$. They described the fountain behaviour as steady but eventually becoming unsteady at the high $R e$ and $F r$ part of their experimental range. The authors also found viscous dissipation to be significant for $R e \lesssim 50$, and the penetration height scales with $Z_{m} / R_{0}=C F r e^{\phi}$ where $\phi \sim 0.5-0.6$. The authors performed additional tests for $60<R e<200$ where the flow becomes more turbulent, and found that the effect of viscosity becomes less important and the scaling for the penetration height approaches the linear relation of Turner (1966). Lin \& Armfield (2004) examined the behaviour of transitional forced fountains for $1 \leqslant F r \leqslant 8$ and $200 \leqslant R e \leqslant 800$ using an axisymmetric numerical simulation. The authors found that the mean height scales like $F r R e^{1 / 4}$. It should be noted that above $R e=200$ the fountains were highly unsteady and the axisymmetric assumptions were probably invalid. 
Zhang \& Baddour (1997) experimentally investigated the behaviour of transitional and turbulent plane fountains with $650<R e<5400$ and $0.62<F r<113$. They found that for low Froude numbers the fountains were relatively stable, with small oscillations in height and little entrainment with the fresh ambient fluid. For $\mathrm{Fr} \gtrsim 5$ the fountains became unstable and greater fluctuations in height were observed. Zhang \& Baddour (1998) observed similar behaviour in turbulent round fountains over the range $850<R e<12750$ and $0.37<F r<36.2$. For $R e=1560$ and $F r=0.94$ the fountain height was steady with little mixing, but fluctuations in height occurred, increasing with Froude number. The authors found that for $\mathrm{Fr}<7$ the mean maximum penetration height no longer scales linearly with $F r$ but is better represented by $Z_{m} / R_{0}=1.7 \mathrm{Fr}^{1.3}$. For $\mathrm{Fr}>7$ they found $Z_{m} / R_{0}=3.06 \mathrm{Fr}$.

Kaye \& Hunt (2006) employed an analytical model to find scaling relations for the initial rise height of weak turbulent/transitional fountains. Using simplifying assumptions for the entrainment of ambient fluid in the rising fountain, the authors developed scaling relations for three regions: forced fountains occurring at $F r \gtrsim 3.0$, weak or intermediate fountains at $1.0 \lesssim F r \lesssim 3.0$ and very weak fountains for $F r \lesssim 1.0$. The authors found $Z_{m} / R_{0} \sim F r$ and $Z_{m} / R_{0} \sim F r^{2}$ for forced and intermediate fountains respectively. For very weak fountains the authors related the radial outflow of fountain fluid from the nozzle to flow over a weir. Using this analogy the authors found $Z_{m} / R_{0} \sim F r^{2 / 3}$, the same Froude number scaling as obtained by Lin \& Armfield $(2000 c)$. Kaye \& Hunt (2006) presented strong experimental evidence supporting these scalings but did not test for any Reynolds number dependence.

While there has been significant coverage given to both weak and forced turbulent fountains, laminar transitional flows are comparatively less well understood. From previous work, it is still not clear what the Reynolds and Froude number dependence of laminar and transitional fountain flow is and the flow behaviour in this region is not well described. In this study we experimentally examine the behaviour of fountains over a wide range of Froude and Reynolds numbers, from weak to strongly forced flow and from laminar to fully turbulent flow. We find the flow to be dominated by several unstable modes in both the laminar and transitional regions. These modes are evident up to what appears to be a fully turbulent limit of $R e=2000$. For weak laminar fountains we identify a number of sub-regions where the behaviour varies significantly with both Reynolds number and Froude number. In all laminar and transitional regions the flow is found to have a Reynolds number dependence.

Our experimental setup is described in $\S 2$. In $\S 3$, the observed fountain behaviour is described and located on a Froude and Reynolds number plane, which forms a regime map. The mechanisms behind the observed behaviour and the ramifications for future scaling relations are discussed in $\S 4$. The conclusions are summarized in $\S 5$.

\section{Experimental setup}

Experiments have been performed by injecting salt water up into a fresh-water tank, $25 \mathrm{~cm}$ deep with $34 \mathrm{~cm}$ square base. The saline water is fed from a header tank to the base of the fresh-water tank as shown in the arrangement in figure 1. The water is injected from a sudden start and maintained at a constant flow rate throughout the experiment. During an experiment the saline fluid rises and then falls back on itself, and finally spreads along the base of the fresh-water tank. The problem is similar to the filling box problem of Turner (1966) and Baines et al. (1990), but in this study the long-term behaviour of the fountain as the tank fills up is not of interest and the experiment is stopped before the saline water intrusion reaches the walls of 


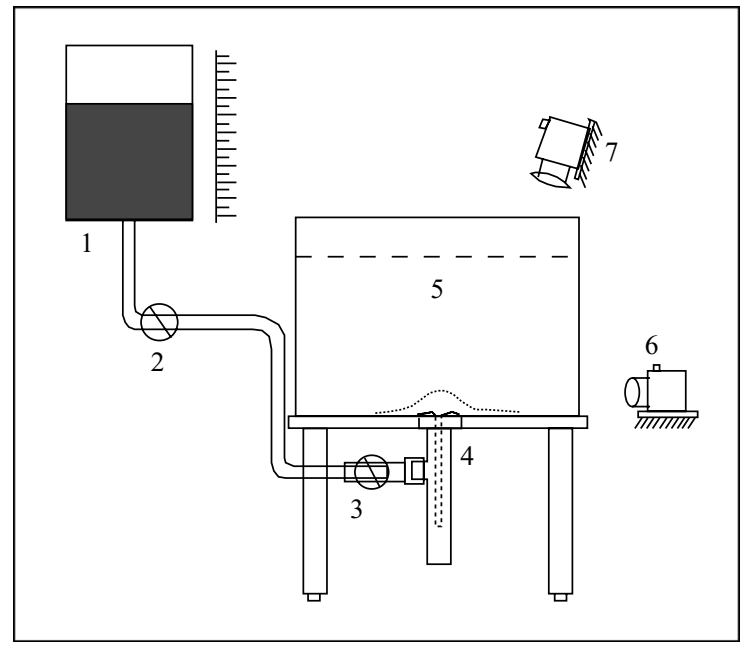

FIGURE 1. Schematic representation of fountain experimental setup with: 1, header tank; 2 , on/off valve; 3 , flow control valve; 4 , inlet nozzle; 5 , fresh-water tank; 6-7, digital cameras.

the tank. Additionally, in this study the inlet pipe is aligned flush with the bottom of the fresh-water tank, unlike many previous investigations (Turner 1966; Baines et al. 1990; Philippe et al. 2005) where a re-entrant nozzle was used. To the authors' knowledge, the only previous studies of round fountains using this configuration are the numerical studies of Lin \& Armfield $(2000 a, c ; 2004)$ and the experimental work of Campbell \& Turner (1989) and Pantzlaff \& Lueptow (1999), although in many previous studies the experimental arrangement is unclear. This configuration may be significant for low-Froude-number weak fountain behaviour, as discussed in $\S 4$.

The fountain inlet flow rate, inlet pipe diameter and the salinity of the inlet fluid were varied to cover a wide range of Reynolds and Froude numbers. The volume flow rate varied from $0.06 \mathrm{~cm}^{3} \mathrm{~s}^{-1}$ to $20 \mathrm{~cm}^{3} \mathrm{~s}^{-1}$. Nozzle diameters of $4.80 \mathrm{~mm}, 2.54 \mathrm{~mm}$, $1.66 \mathrm{~mm}, 1.13 \mathrm{~mm}, 0.76 \mathrm{~mm}$ and $0.54 \mathrm{~mm}$ were used. The ratio of inlet pipe length to pipe diameter was greater than 40 in each case, ensuring that the flow is fully developed as it enters the tank. The density ratio is varied with $0.004<\Delta \rho / \rho_{f}<0.16$, where $\Delta \rho=\rho_{0}-\rho_{f}$. The salinity also effects the solution viscosity and in this work it has values $1.01 \times 10^{-6}<v_{0}<1.4 \times 10^{-6} \mathrm{~m}^{2} \mathrm{~s}^{-1}$. The maximum ratio of the viscosity of the ambient fluid and the inlet fluid is therefore $v_{0} / v_{f} \leqslant 1.4$.

Small concentrations of dye were used as the tracer in the saline water. The effect of the dye on the solution density was taken into account. The flow was recorded on two digital cameras at 15 frames per second, with experiments running for less than 2 minutes or until the saline intrusion front reached the edge of the tank. For the highest-Reynolds-number cases in this study $(R e>1000)$, the flow rate at the source was very high and the saline intrusion formed very rapidly. In order to allow turbulent flow to be examined in this small experimental rig over a reasonable time period, in these cases, the saline intrusion was allowed to reach the tank walls and rise through the run forming a shallow layer. This layer depth is, however, very small compared to the height of the fountain.

The Schmidt number, given by $S c=v_{0} / D$, where $D$ is the binary diffusion coefficient for salt in water, is about 700 in these experiments. Molecular diffusion is therefore unlikely to be an important factor here. We note, however, that in other applications 


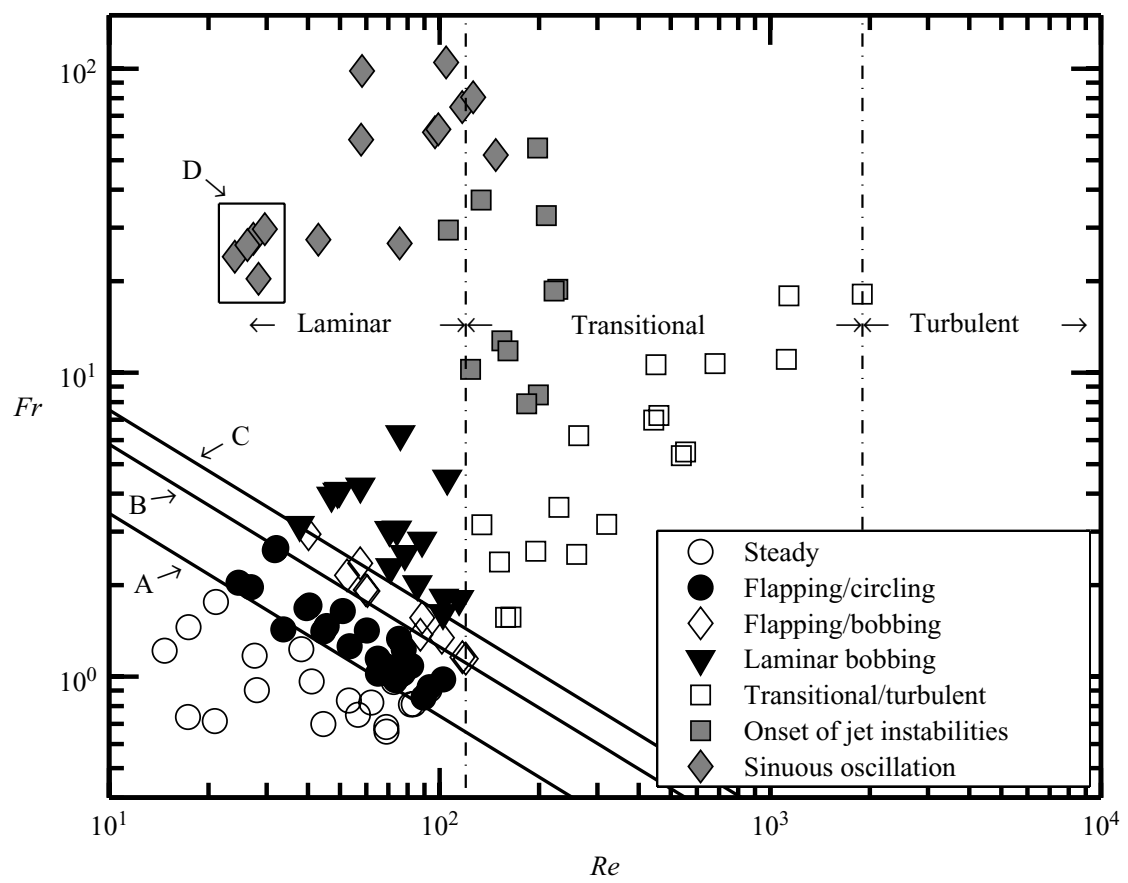

FIGURE 2. Regime map of fountain behaviour with $\operatorname{Re}$ and $\mathrm{Fr}$. Solid lines $\mathrm{Fr} R e^{2 / 3}=C$ where the constant $C$ is: A, $16 ; \mathrm{B}, 27 ; \mathrm{C}, 35$.

such as thermal fountains, particularly for gases, molecular diffusion is greater and may be a significant factor.

\section{Observations}

The experiments were performed over the ranges $0.7<F r<100$ and $15<R e<1900$, and the observed fountain behaviour was placed on the regime map of Froude and Reynolds numbers in figure 2. Broadly, the flow can be categorized as laminar for $R e<120$, with the transition to turbulence occurring above this region independent of Froude number. No turbulence statistics were taken so these observations are purely visual. Within both the laminar and transitional regions, a number of sub-groupings have been made, which are described below.

In the laminar region for $F r<10$, a range of behaviour is observed that has both Reynolds and Froude number dependence. The flow has been categorized into steady, flapping/circling, flapping/bobbing and laminar bobbing regions. The demarcation lines between different laminar fountain behaviour are found to be well described by $C=F r e^{2 / 3}$ for $10<\operatorname{Re}<120$ and $0.7<F r<10$, as shown in figure 2 .

The laminar steady region is observed for $0<F r<16 R e^{-2 / 3}$ over the range of Reynolds numbers examined in this study. In this region the flow is stable and axisymmetric with no fluctuation in fountain height, and is similar to the weak fountains examined by Lin \& Armfield (2000a,c). A typical visualization of a weak steady fountain is given in figure 3. Here and in figure 4 , the contrasting refractive index of the saline and fresh water is used to visualize the flow rather than dye which has been used in the subsequent images. These images were taken by blocking all light from the tank and using flash photography, where the flash was situated adjacent to the camera. The saline intrusion can be seen in this image as the interface between saline and fresh water is lifted slightly from the bottom of the tank. 


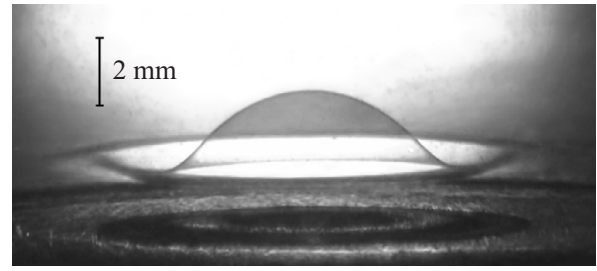

FIGURE 3. Visualization of a weak steady axisymmetric fountain at $R e=21$ and $F r=0.71$, with $U_{0}=0.88 \mathrm{~cm} \mathrm{~s}^{-1}, R_{0}=2.4 \mathrm{~mm}$ and $\Delta \rho / \rho_{f}=0.00646$.
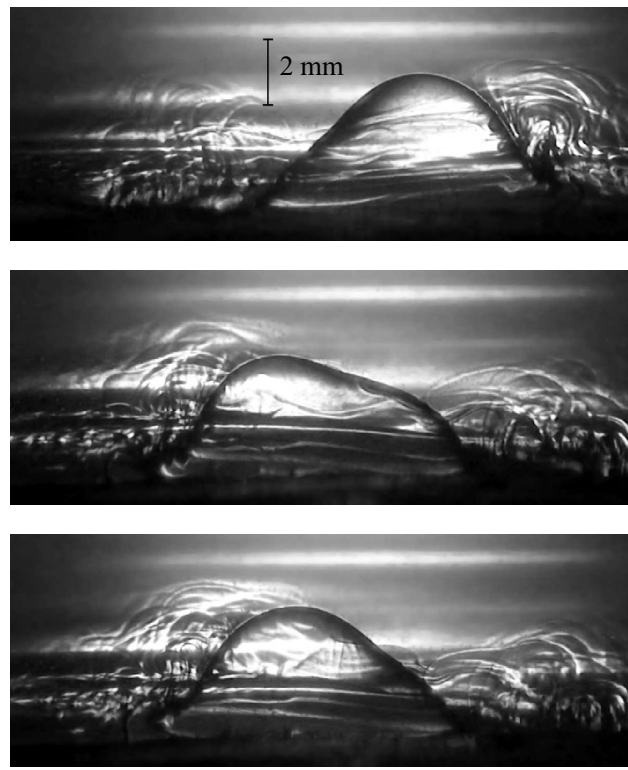

FIgURE 4. Visualization of flapping fountain at $R e=72$ and $F r=1.0$, with $U_{0}=3.2 \mathrm{~cm} \mathrm{~s}^{-1}$, $R_{0}=2.4 \mathrm{~mm}$ and $\Delta \rho / \rho_{f}=0.00454$.

Between $16 R e^{-2 / 3}<F r<27 R e^{-2 / 3}$ the flow becomes unstable, resulting in several interesting modes of oscillation. The first mode of oscillation is a two-dimensional flapping motion. This usually, but not always, develops into a flapping mode, whereby the plane of flapping rotates about the fountain axis, so the direction of flapping changes. This behaviour then develops into a circling or multimodal flapping motion, where the top of the fountain circles around the fountain axis. In many cases the fountain was observed to switch continually between the different flapping/circling modes. The motion of a flapping fountain is captured in figure 4 , where the peak of the fountain can been seen as it moves from one side to the other. The motion can also be seen in movie 1 (available with the online version of the paper).

In the laminar bobbing region the fountain behaviour is highly unsteady and threedimensional. It is characterized by the rapid fluctuation in fountain height, as the fountain front continually rises, stagnates and then collapses around the next rising front. A distinct difference between this behaviour and the flapping/circling modes is that, with the bobbing motion, the fountain height is always at a maximum in the centre of the fountain axis, whereas for the flapping or circling modes, the location of maximum fountain height is always off-axis. On the regime plot in figure 2, this region occurs for $35 R e^{-2 / 3}<F r \lesssim 10$. A series of typical images of a laminar bobbing 
(a)

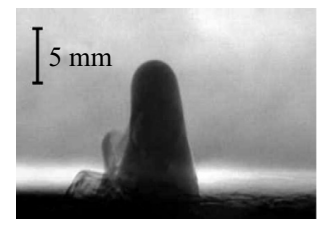

(d)

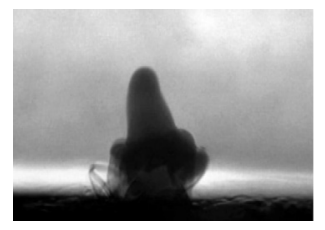

(g)

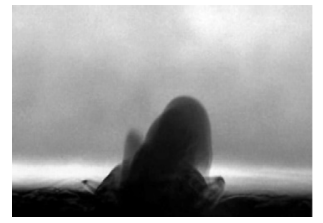

(j)

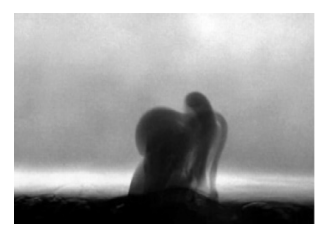

(m)

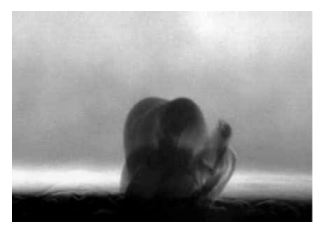

(p)

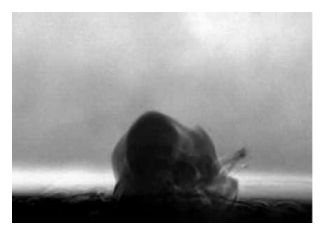

(b)

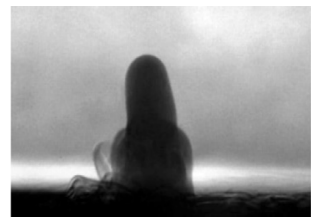

(e)

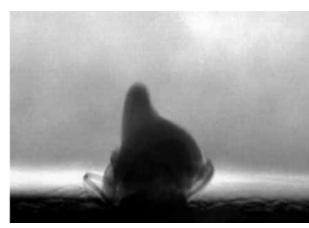

(h)

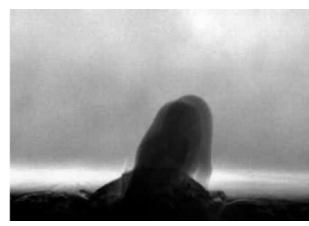

(k)

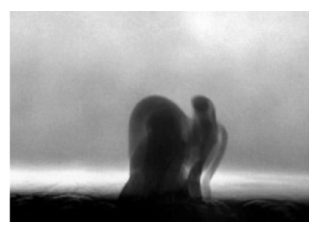

(n)

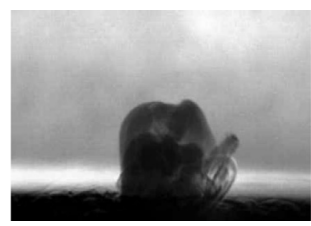

(q)

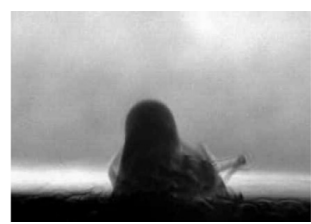

(c)

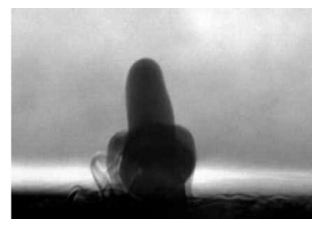

(f)

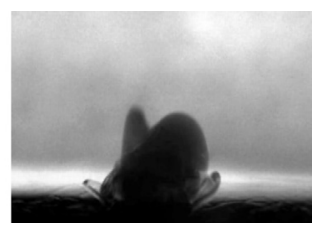

(i)

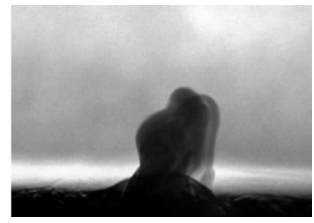

(l)

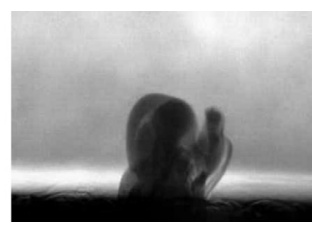

(o)

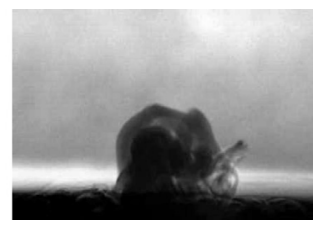

(r)

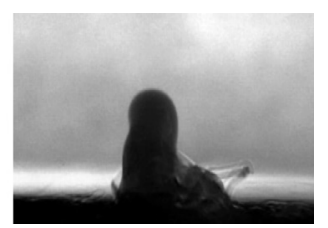

FIGURE 5. Visualization of laminar bobbing behaviour present at $R e=123$ and $F r=2.1$, with $U_{0}=5.4 \mathrm{~cm} \mathrm{~s}^{-1}, R_{0}=2.4 \mathrm{~mm}$ and $\Delta \rho / \rho_{f}=0.033$. Between $(a)$ and $(r)$ a series of fountain fronts rise and fall around the fountain column. Images are about $60.7 \mathrm{~ms}$ apart, with image (a) taken $12.991 \mathrm{~s}$ from the start of the run.

fountain is given in figure 5. In figure $5(a)$ the fountain is at its maximum height. In figure $5(a-f)$ the fountain front collapses and then rises again in figure $5(g-k)$, collapses in figure $5(l-o)$ and then rises again in $(p-r)$. Between the flapping motion 
and the bobbing motion there is a transition region for $27 R e^{-2 / 3}<F r<35 R e^{-2 / 3}$, where both behaviours can be observed alternately or together. This has been labelled flapping/bobbing in figure 2. This behaviour is shown in movie 2 (available with the online version of the paper).

At higher Froude numbers $(F r \gtrsim 10)$ in the laminar region $(20<R e<120)$, the fountain behaviour appears to be fundamentally different. Instead of collapsing as in the bobbing region, the fluid column rises to a much greater height in a steady stable manner. The flow is characterized by a broad fountain head, where the stagnating fountain fluid is spread horizontally a short distance before reversing back on the rising column, partially enveloping the ambient fluid. Instead of collapsing on the rising column like the laminar bobbing flow, the reversing fluid flows down around the rising column.

For all the points in the region defined by $20<R e<120$ and $F r \gtrsim 10$, the interaction between the descending fluid and the rising fluid column appears responsible for several other interesting phenomena. At low Reynolds number an intermittent sinuous instability is observed, appearing as a wave travelling up the fluid column. This leads to a slight wobbling of the fountain head. In the high-Reynolds-number part of this range, the sinuous instability is manifested more strongly. When the waves reach the head of the fountain and the stagnating/reversing fluid, the amplitude increases and the column appears to periodically buckle at the head. This leads to an increase in flow falling around this rising column and a corresponding increase in shear. This sometimes, but not always, results in partial collapse of the fountain. A typical example of this behaviour is shown in figure 6 for flow at $R e=105$ and $F r=105$. Here the sinuous wave is clearly visible in the fluid column. At the head of the fountain, a large region of stagnating dense fluid forms, from which fluid then descends around the fluid column in an unsteady manner. This behaviour is shown in movie 3 (available with the online version of the paper).

A peculiar and very interesting phenomenon occurs in the low-Reynolds-number part of this region. The fluid falling back towards the source flows in a laminar cylindrical sheet entirely enveloping the rising fluid column and trapping ambient fluid inside. This trapped fluid is entrained in the descending flow and is slowly advected out of the fountain. This entrainment is aided by the sinuous motion of the fluid column and surrounding enveloping sheet. Additionally, the trapped fluid can, in the initial stages of the flow, be ejected suddenly by the partial collapse of the enveloping sheet. The collapse events appear to be related to the sinuous motion in the fountain. This motion can be seen in movie 4 (available with the online version of the paper). As the volume of trapped fluid decreases, the rising and falling fluid come in direct contact. The shear force acting on the rising column is then increased and the fountain height is then reduced. The runs in which this behaviour has been observed are indicated in figure 2 by the region marked $D$. The process is depicted in figure 7. For the first 30 seconds, the descending fluid rises and then falls around the fountain source before entirely enveloping the ambient fluid. Between $30 \mathrm{~s}$ and $50 \mathrm{~s}$ some volume of fluid is ejected by a partial collapse of the enveloping flow. After $120 \mathrm{~s}$ the fluid has been entirely advected out and the fountain penetration height is reduced. The time scale for the advection appears to be related to the diffusion of momentum. If the transport of the trapped fluid, represented by the scalar $\phi$, can be written as

$$
\frac{\partial \phi}{\partial t} \approx v \frac{\partial^{2} \phi}{\partial x^{2}},
$$




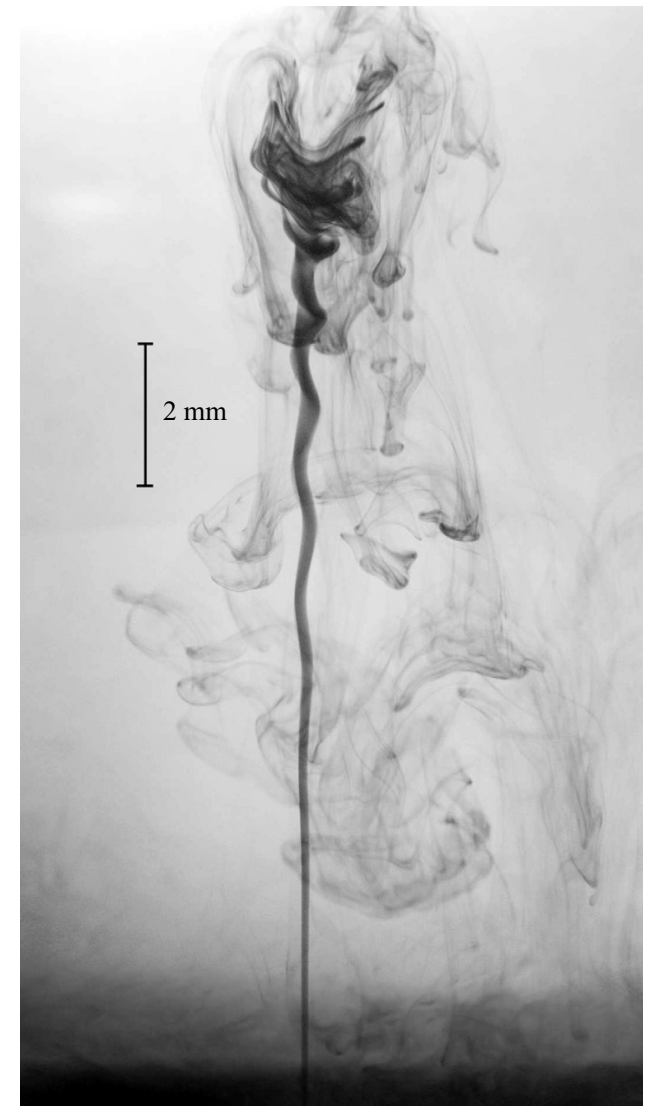

FIGURE 6. Visualization of sinuous behaviour present at $R e=105$ and $F r=105$, with $U_{0}=27.6 \mathrm{~cm} \mathrm{~s}^{-1}, R_{0}=0.38 \mathrm{~mm}$ and $\Delta \rho / \rho_{f}=0.00186$.

where $v$ is the fluid kinematic viscosity, then $t \sim x^{2} / \nu$. With a length scale of $x \sim 1 \times$ $10^{-2} \mathrm{~m}$ (fountain height in figure 7), a viscosity of $v=1 \times 10^{-6} \mathrm{~m}^{2} \mathrm{~s}^{-1}$, this gives a time scale for diffusion of $t \approx 100 \mathrm{~s}$, the same as the time scale found from the experiment as shown in figure 7 .

In the same range of Froude numbers $(F r \gtrsim 10)$, as the Reynolds number is increased into the transitional region shown in figure 2, the sinuous instability appears to intermittently break down into a turbulent jet-like flow at some point along the rising fluid column. The location of this point moves up and down the rising fluid column and, as with the sinuous instability in the laminar region, this appears to be initiated by a shear interaction between the rising column and the descending fluid. This instability results in major fountain collapse events leading to large oscillations in the fountain penetration height. This instability is most clear in the points grouped as 'Onset of jet instabilities' in figure 2, where the flow is not very turbulent and both the sinuous instabilities and the jet-like flow breakdown are observed together. Two visualizations of this flow behaviour are presented here. The first is in figure 8 , at $R e=105$ and $F r=105$, the same as in figure 6 . Here the sinuous instability is very strong but the jet instability occurs only intermittently. The instability is marked by sudden oscillation of flow at a localized segment of the column. This wave then rises up the column, increasing in length. At this Reynolds number, the instability does 
(a)

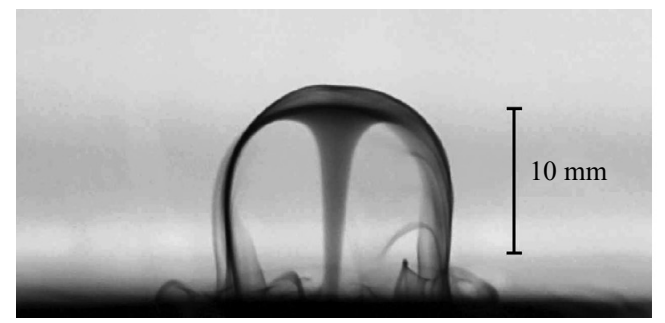

(c)

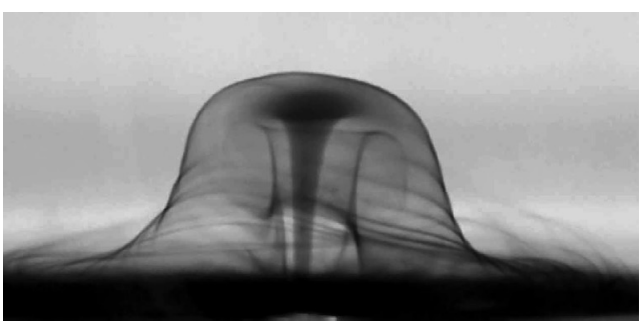

(e)

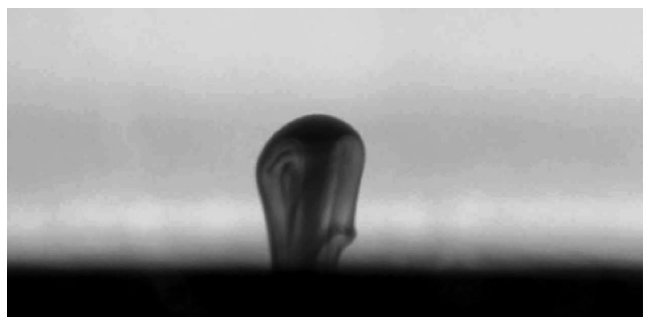

(b)

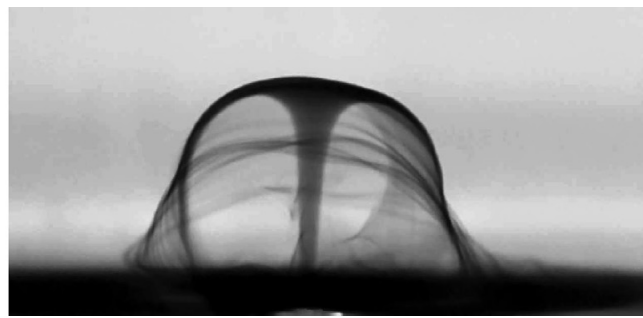

(d)

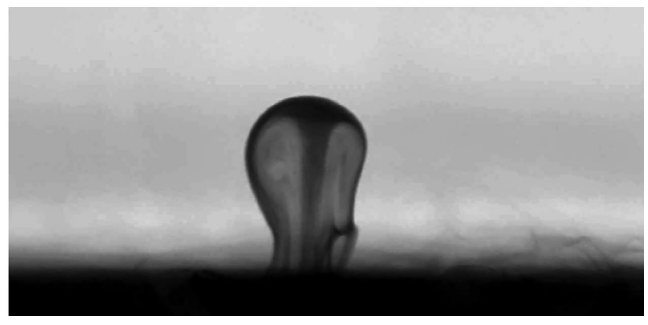

(f)

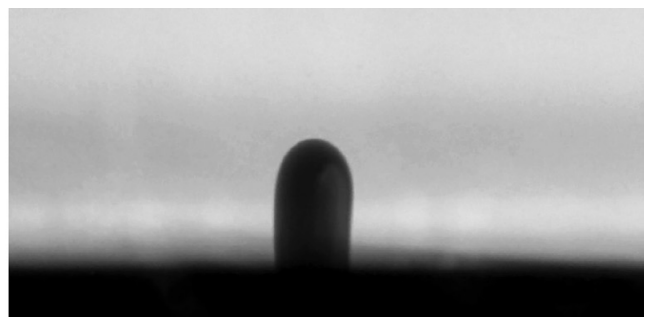

FIGURE 7. Visualization of fountain behaviour at $R e=26$ and $F r=26$, with $U_{0}=6.9 \mathrm{~cm} \mathrm{~s}^{-1}$, $R_{0}=0.38 \mathrm{~mm}$ and $\Delta \rho / \rho_{f}=0.00186$. Images $(a)-(f)$ were taken $10 \mathrm{~s}, 20 \mathrm{~s}, 30 \mathrm{~s}, 50 \mathrm{~s}, 90 \mathrm{~s}$ and $120 \mathrm{~s}$ after the start of the run, respectively.

not cause the fountain to collapse and eventually dies out. The second set of images is given in figure 9 for $R e=221$ and $F r=18$. Here, the buoyancy forces are stronger and the jet-like breakdown occurs periodically and results in fountain collapse, as shown in the sequence. In figure $9(a)$ the flow is laminar and is at the maximum penetration height. In figure $9(b)$ the instability is triggered at some point along the fountain column. This rapidly spreads throughout the column, leaving partially turbulent jet-like flow at the fountain source. In figure $9(c)$ the instability dies out and the flow re-laminarizes, rising through the collapsed fountain flow as shown in figure $9(d)$. A short time later the sequence is repeated. This behaviour can also be seen in movie 5 (available with the online version of the paper).

For $F r \lesssim 10$ in the transition region, there is also strong oscillatory behaviour but the jet-like instabilities become more difficult to visualize. An example of the behaviour observed at $R e=183$ and $F r=8$ is shown in figure 10 . Here the sequence demonstrates how the flow rises, reaches its peak, and then, as descending fluid flows back around the rising column, some mechanism causes the fountain to collapse. The nature of this mechanism is difficult to explain from the images alone. It is clear, however, that as the Froude number is decreased, the shear-driven instabilities and jet-like behaviour are less evident and at $F r \lesssim 5$ appear to be entirely absent. This 
(a)

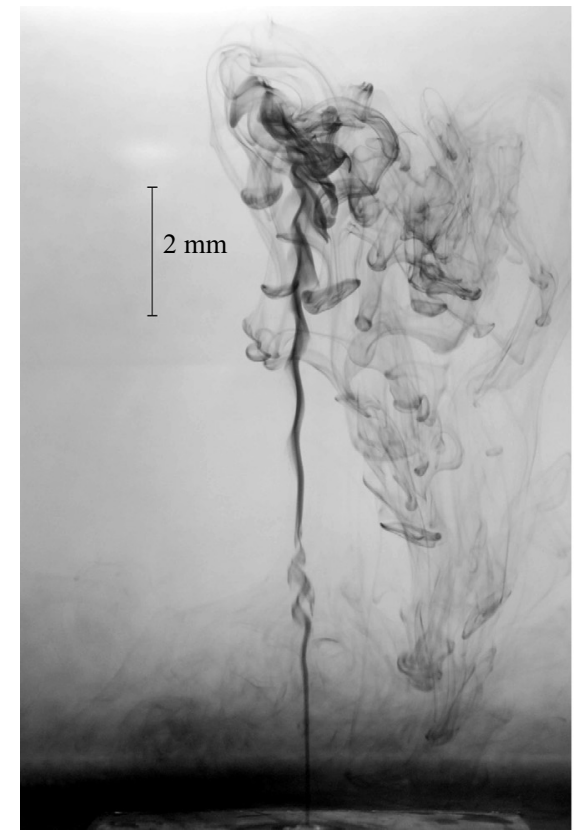

(b)

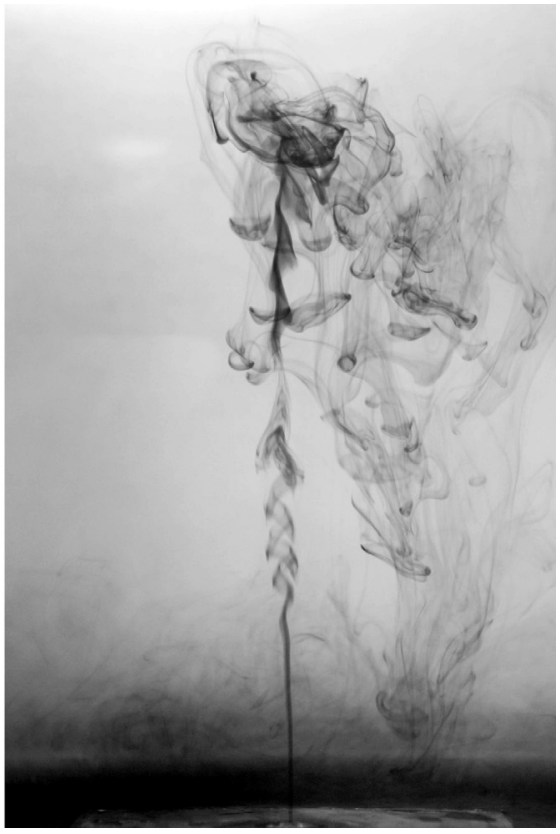

FIGURE 8. Visualization of sinuous instability breaking down into jet-like behaviour for $R e=105$ and $F r=105$, with $U_{0}=27.6 \mathrm{~cm} \mathrm{~s}^{-1}, R_{0}=0.38 \mathrm{~mm}$ and $\Delta \rho / \rho_{f}=0.00186$. Images $(a)$ and $(b)$ were taken $50 \mathrm{~s}$ and $51 \mathrm{~s}$ after the start of the run, respectively.

(a)

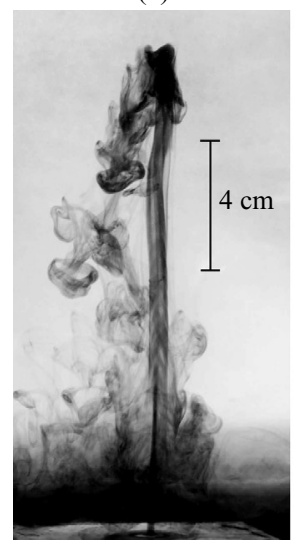

(b)

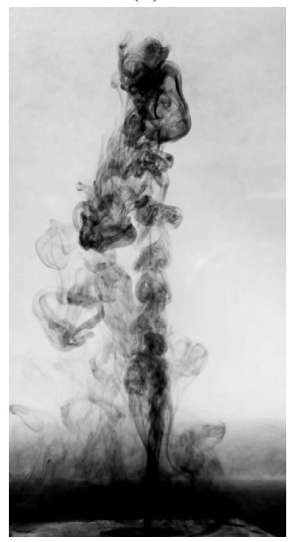

(c)

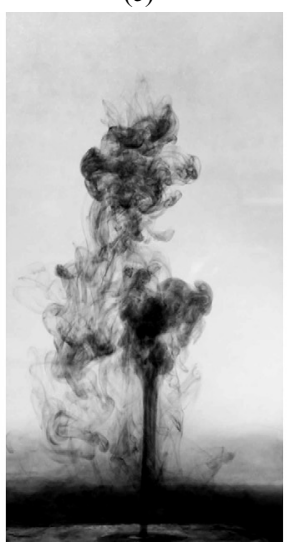

$(d)$

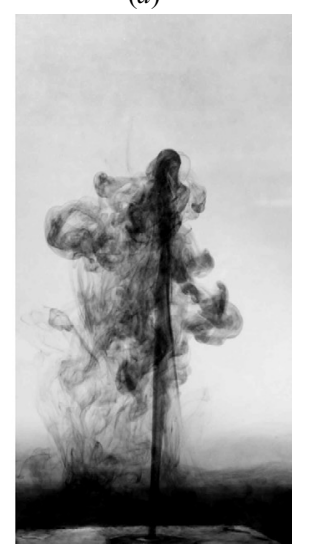

FIGURE 9. Visualization of jet breakdown behaviour at $R e=221$ and $F r=18$, with $U_{0}=17.7 \mathrm{~cm} \mathrm{~s}^{-1}, R_{0}=1.27 \mathrm{~mm}$ and $\Delta \rho / \rho_{f}=0.00727$. Images $(a)-(d)$ were taken $1 \mathrm{~min} 26 \mathrm{~s}$, $1 \mathrm{~min} 27 \mathrm{~s}, 1 \mathrm{~min} 29 \mathrm{~s}$ and $1 \mathrm{~min} 31 \mathrm{~s}$ after the start of the run, respectively.

low-Froude-number transitional behaviour is similar to the laminar bobbing, where the fountain continually rises, stagnates and then falls back to the source around new rising flow. There are similarities between this action and the behaviour described by Clannet (1998) of a water fountain injected into air. In that study the author observed that the water would rise, then, under the action of gravity, slow and eventually stall, 
(a)

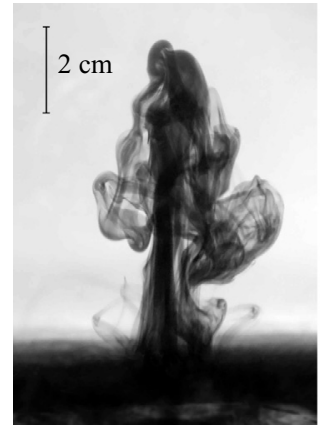

(d)

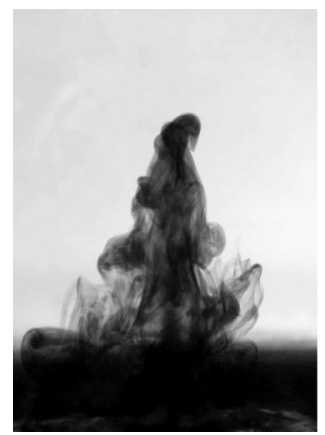

(b)

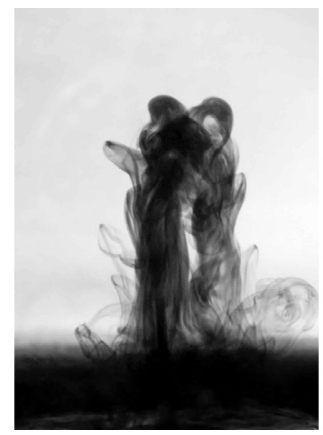

(e)

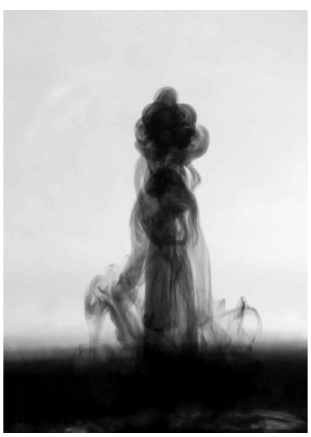

(c)

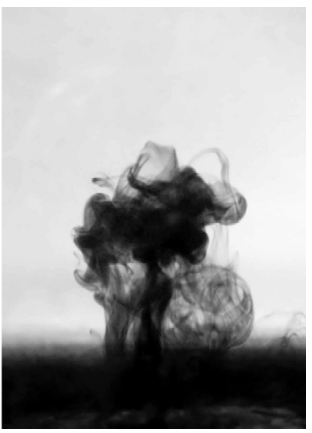

$(f)$

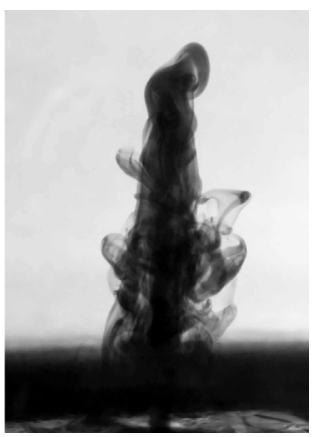

FIGURE 10. Visualization of transitional behaviour for $R e=183$ and $F r=8$, with $U_{0}=$ $7.7 \mathrm{~cm} \mathrm{~s}^{-1}, R_{0}=2.4 \mathrm{~mm}$ and $\Delta \rho / \rho_{f}=0.00403$. Images $(a)-(f)$ were taken $1 \mathrm{~min} 41 \mathrm{~s}, 1 \mathrm{~min}$ $44 \mathrm{~s}, 1 \mathrm{~min} 46 \mathrm{~s}, 1 \mathrm{~min} 48 \mathrm{~s}, 1 \mathrm{~min} 49 \mathrm{~s}$ and $1 \mathrm{~min} 53 \mathrm{~s}$ after the start of the run, respectively.

forming a lump of mass at the top of the fountain. This lump would then fall back to the source and then dislodge, allowing a new jet to rise. While surface tension effects may be important in this flow, it does provide a simple explanation for the phenomena observed here. The lowest Froude number in the transitional region tested in this study is $\mathrm{Fr}=1.5$. Friedman et al. (2007) experimentally examined turbulent weak round fountains, using glycerin/water mixtures for the fountain fluid and silicon oil for the ambient fluid, over the range $90<R e<6000$. The authors found that at a critical Froude number of $F r \approx \sqrt{2}$ the fountain flow becomes unstable. While the ambient and fountain fluids are immiscible in their study, the work is relevant to the present study, as it demonstrates that across a very wide range of Reynolds numbers, buoyancy forces can stabilize the flow completely at low Froude numbers.

Beyond $R e \approx 400$, the flow becomes more turbulent with stronger mixing and entrainment of ambient fluid. Some form of periodic collapse and rise behaviour of the fountains has been observed clearly in most of the transitional/turbulent region, up to $R e \approx 1000$. Beyond $R e \approx 1000$ the oscillatory behaviour is less distinct. At $R e=1900$, the highest Reynolds number tested in this study, the flow behaviour is much closer to the descriptions and images provided by Turner (1966) and Pantzlaff \& Lueptow (1999). The flow starts strongly as a jet, reaches its peak height and then quickly settles into a stable turbulent fountain flow with a penetration height of approximately $70 \%$ of the peak height. The only oscillations present are small highfrequency pulses that can be expected in turbulent flow. 


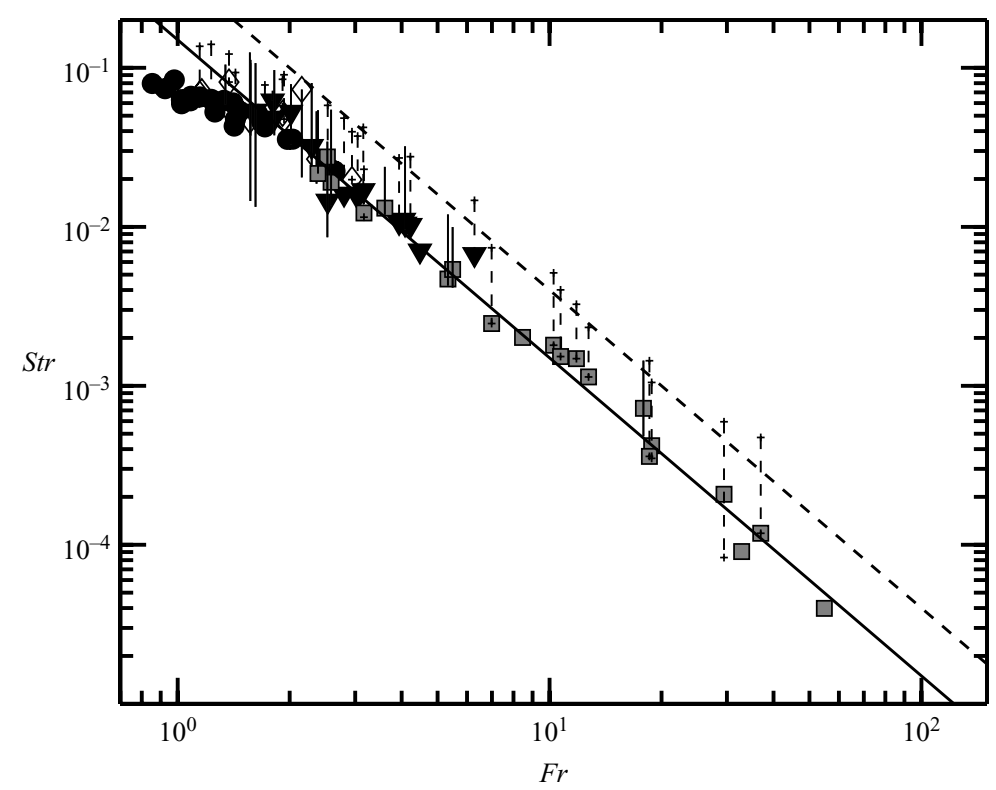

FIGURE 11. Strouhal number for primary mode of oscillation in height, marked with symbols defined in figure 2. Solid black vertical lines indicate range of frequencies present for multimodal flows. Limits of dashed vertical lines indicate secondary frequencies if present. Thick solid line: $S t r=0.15 / F r^{2}$, thick dashed line: Str $=0.4 / F r^{2}$.

\section{Discussion}

The spectrum of frequencies from the time series of fountain height shows in most cases a single dominant mode of oscillation. These dominant modes have been plotted in figure 11, as Strouhal number against Froude number where the Strouhal number is $\operatorname{Str}=f R_{0} / U_{0}$ and $f$ is frequency in Hz. Where secondary frequencies are observed, these have been marked on the plot. For the flapping and circling cases, this frequency corresponds to that of the flapping/circling. For the fountains at Froude and Reynolds numbers grouped as 'Onset of jet instabilities', 'Transitional/turbulent' or as 'Laminar bobbing' in figure 2, the primary frequency corresponds to the frequency of major fountain collapse. The flapping/bobbing transition region is characterized by multimodal flapping/bobbing, and picking a dominant mode is very subjective. In these regions the range of frequencies present are marked in figure 11 by dark lines. The cases marked as 'Sinuous oscillation' have been excluded, as the oscillation in height in this region is unclear and irregular.

The dominant modes for the laminar bobbing flow and the flow in the transitional/turbulent region all collapse onto a single line of $\operatorname{Str}=c / F r^{2}$ with $c=0.15$. The flapping and transitional flapping/bobbing fountains appear to depart from the $\mathrm{Str} \sim 1 / \mathrm{Fr}^{2}$ line at low Froude numbers. In this region the curve appears to approach a line of constant Strouhal number. Friedman (2006) noted that above $\mathrm{Fr} \sim 1$, round fountains (using a re-entrant nozzle) become unstable with the fountain rising and collapsing in a periodic manner. The author found that this behaviour was well described by a line of constant Strouhal number, Str $=0.10$ for $1.0<F r<3.16$. For $\mathrm{Fr}>3.16$ the fountain behaviour was noted to be more unpredictable, with a less consistent frequency of oscillation. Preliminary numerical and experimental results performed by the present authors have shown that the flapping modes observed in 

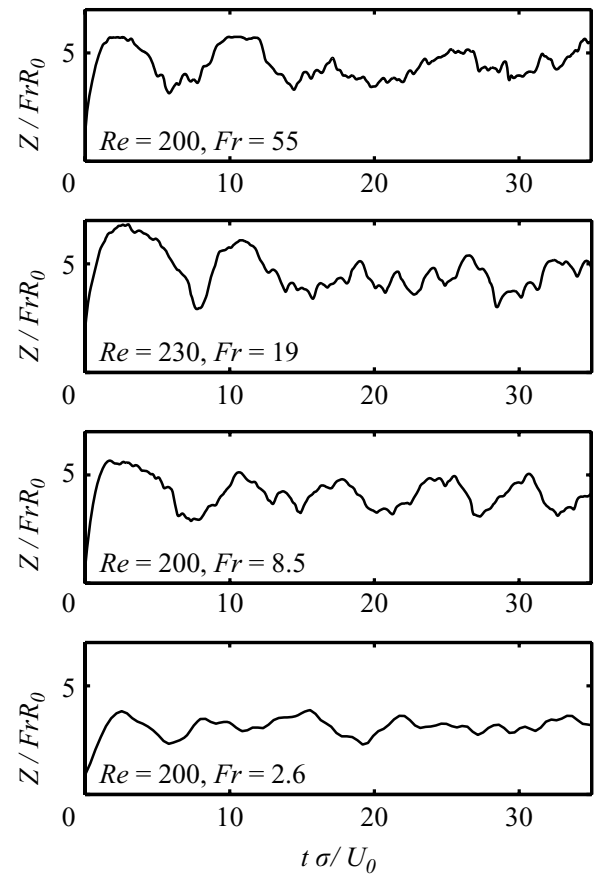

FIGURE 12. Time series of fountain height in transitional region at $R e \sim 200$ and $F r=2.6-55$.

this study only occur for flush wall-mounted nozzles, not re-entrant nozzles. This suggests that this behaviour may be related to the presence of a bounding wall or the saline water intrusion.

In another study, Clannet (1998) found the period of oscillation of water fountains in air could be described by $\operatorname{Str}=1 /\left(3 F r^{2}\right)$. Middleton (1975) was the first to note that the rise time $t$ in starting plumes can be non-dimensionalized using $t \sigma / U_{0}$. This time scaling is equivalent to the scaling for the frequency found here, $S t r \sim 1 / \mathrm{Fr}^{2}$, since

$$
S t r=\frac{f R_{0}}{U_{0}} \sim \frac{1}{F r^{2}}=\frac{\sigma}{U_{0}} \frac{R_{0}}{U_{0}} .
$$

Pantzlaff \& Lueptow (1999) found this scaling applied well to the initial transient stage of the turbulent fountains. With the constants for $c$ given in figure 11, the dimensionless oscillation period of the bobbing and transitional fountain flow is in the range $2.5-6.7 t \sigma / U_{0}$.

The time series of the fountain penetration height, $Z$, is shown in figures $12-15$, for a range of Froude and Reynolds numbers. In figure 12 the effect of Froude number in the transitional region is shown for $F r=2.6-55$ at $R e \sim 200$. The oscillatory behaviour is very clear. Other frequencies are clearly present but the low frequency signal, with, the same period as the initial rise and collapse event, dominates the flow. Interestingly, the low-Froude-number bobbing has the same non-dimensional frequency as the highFroude-number oscillation. The linear Froude number scaling for the fountain height is not good, with clear differences between $F r=2.6$ and $F r=8.5$. In figure 13 typical results for flow in the transitional region at $F r \sim 11$ and $R e=154-1120$ and at $F r=18$ and $R e=1140$ and 1900 are shown. The oscillatory behaviour is strongly present up to $R e=680$, but is very weak at $R e \gtrsim 1120$ and completely absent at $R e=1900$. The 

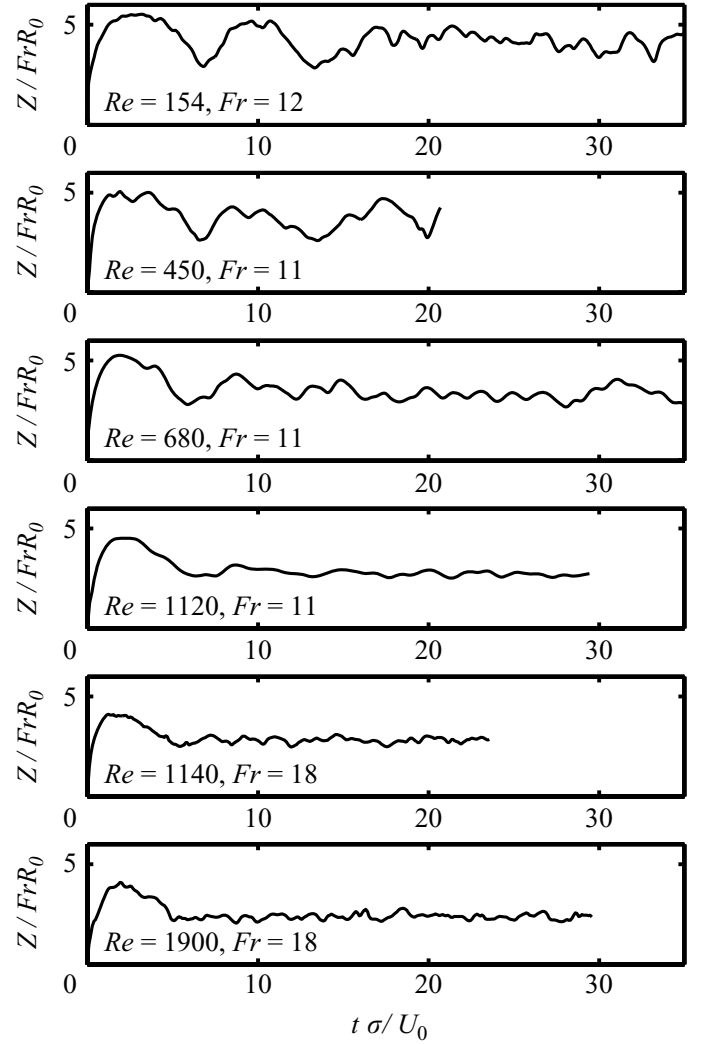

Figure 13. Time series of fountain height in transitional region at $\mathrm{Fr} \sim 11$ and $R e=154-1120$ and at $F r=18$ and $R e=1140$ and 1900 .

three higher-Reynolds-number results compare well with the time series of fountain heights provided in Turner (1966) and Pantzlaff \& Lueptow (1999). Increasing the Froude number from 11 to 18 does not appear to result in an increase in oscillatory behaviour or to change the character of the flow.

In figure 14 examples of flow in the laminar bobbing and transitional region at $F r \sim 4$ and $R e=230-58$ are shown, together with a result in the flapping/bobbing region at $R e=60$ and $F r=1.9$. The flow in the turbulent region for $R e>120$ and the laminar bobbing cases shown at $R e=58$ and 105 both have strong oscillatory behaviour. The dominant non-dimensional bobbing frequency appears to be the same in both the laminar and turbulent regions. The flapping/bobbing mode at $R e=60$ and $F r=1.9$ compares well with the bobbing behaviour, but contains a wider range of frequencies than the other modes.

In figure 15 , the time series for the fountain height of a circling fountain at $R e=44$ and $\mathrm{Fr}=1.4$ is given. Here the fountain height is defined as the highest point in the fountain at any location. The time series for the radial location of this point, as seen in the plane of view of the camera, is also shown in the figure. The fountain height varies very little but the location of maximum height oscillates significantly, regularly about the source.

The mean fountain heights for all the experiments in this study are plotted in figure 16 against Froude number, with the high-Reynolds-number scaling of Turner (1966), the two low-Froude-number scaling relations of Kaye \& Hunt (2006) and 

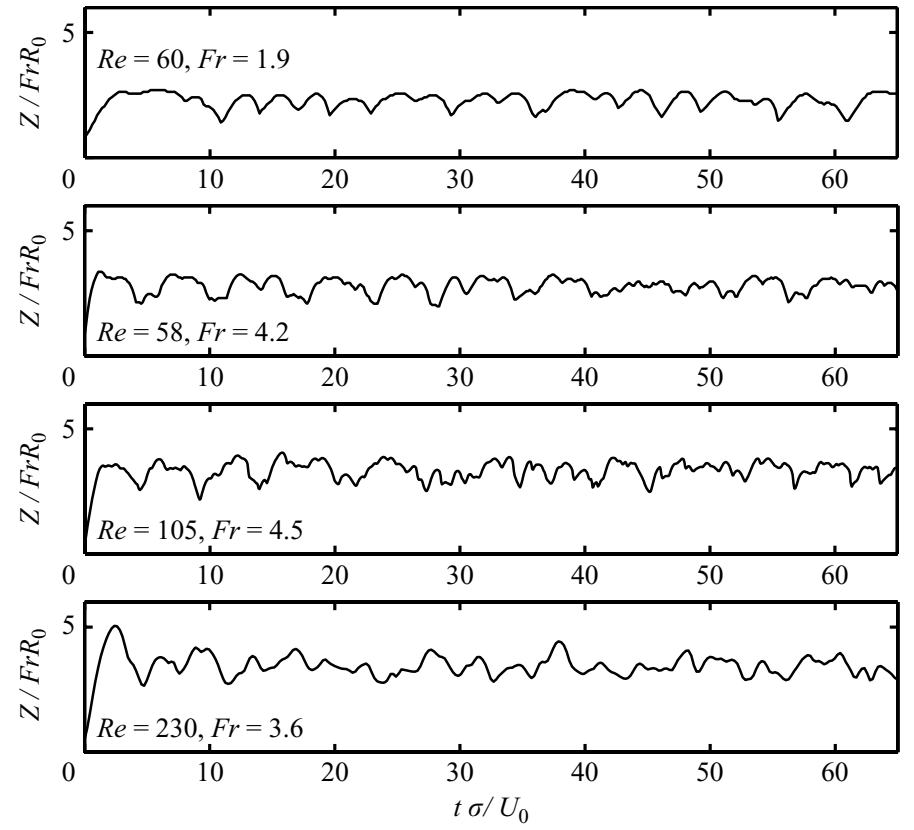

FiguRE 14. Time series of fountain height in the laminar bobbing and transitional region at $F r \sim 4$ and $R e=58-230$. Flapping/bobbing at $R e=60$ and $F r=1.9$ is also shown for comparison.
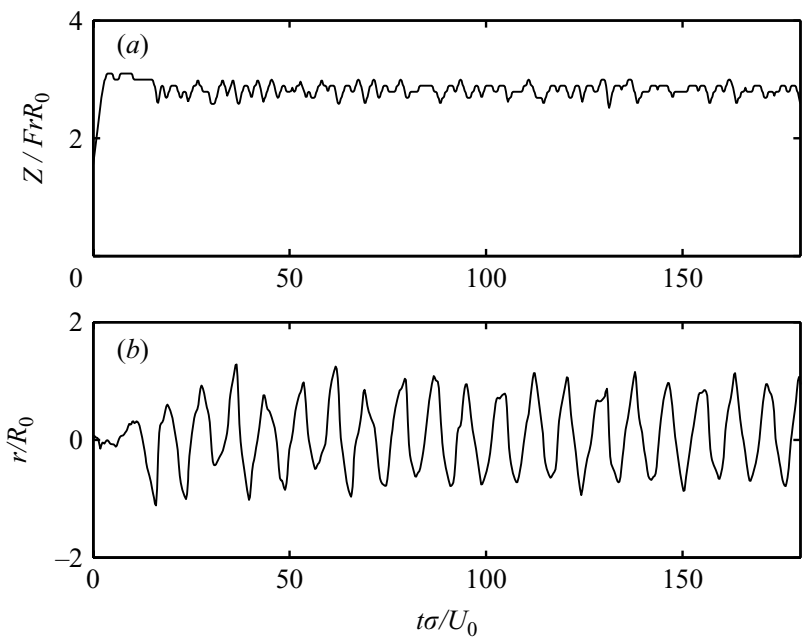

FIGURE 15. Time series of fountain height $(a)$ and fountain centre location $(b)$ for flapping fountain at $R e=44$ and $F r=1.4$.

the two relations of Zhang \& Baddour (1998). For the high-Reynolds-number cases, the mean height is plotted, excluding the initial peak at startup. For the other cases, where strong oscillatory behaviour is observed, the maximum penetration height of each oscillation is used. The same procedure was used by Zhang \& Baddour (1998). The figure shows that none of the previous relations is a very good fit to the data. The poor fit of the low-Froude-number relations of Kaye \& Hunt (2006) is expected, 


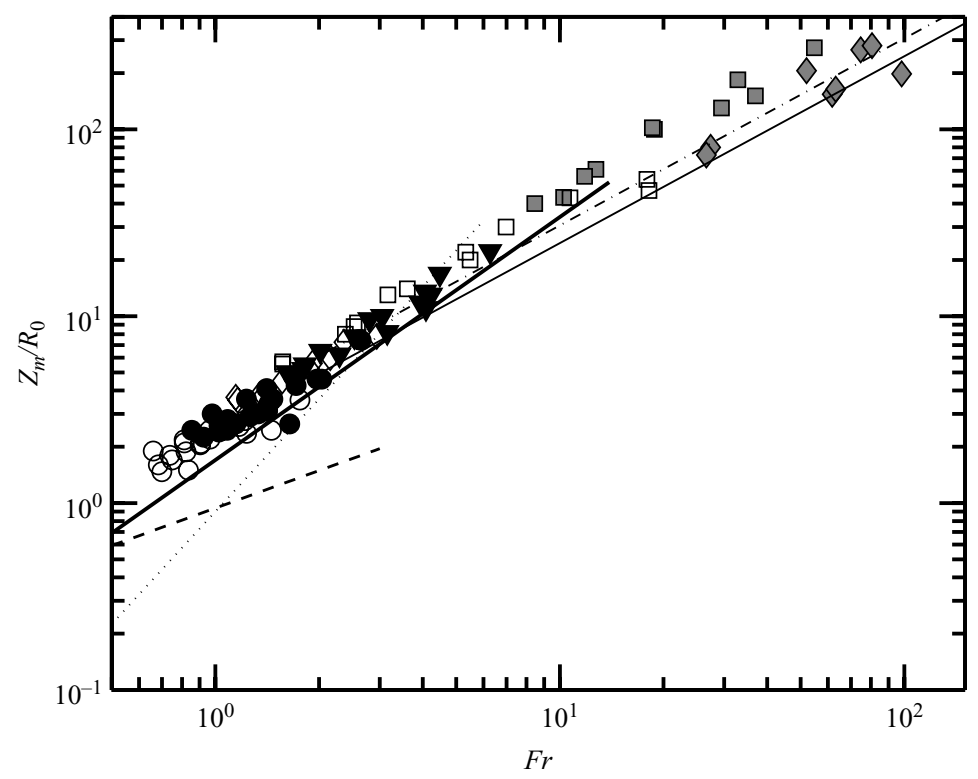

FIGURE 16. Mean maximum penetration height as function of Froude number. Symbols defined in figure 2. Dashed line, $0.94 \mathrm{Fr}^{2 / 3}$ (Kaye \& Hunt 2006); dotted line, $0.9 \mathrm{Fr}^{2}$ (Kaye \& Hunt 2006); thin solid line, $2.46 F r$ (Turner 1966); thick solid line, $1.7 F^{1.3}$ (Zhang \& Baddour 1998); thin dash-dotted line, 3.06Fr (Zhang \& Baddour 1998).

given that the low-Froude-number results in the present study were obtained only at very low Reynolds number, while the low-Froude-number results in Kaye \& Hunt (2006) and also in Zhang \& Baddour (1998) are in the transitional to fully turbulent range. The lower tank boundary wall may also have some influence on the flow. Neither Kaye \& Hunt (2006) or Zhang \& Baddour (1998) specify the nozzle type used.

The two previous studies on laminar and transitional fountains, Philippe et al. (2005) and Lin \& Armfield (2004), found the scaling for the mean fountain height is still linear in the Froude number but contains an additional Reynolds number dependence. The functional dependence of the linear-Froude-number scaling on Reynolds number for the results in this study can be seen in figure 17 where the quantity $Z_{m} / F r R_{0}$ is plotted against Reynolds number. The high-Froude-number runs with $R e<300$ are reasonably well fitted by $Z_{m} / \operatorname{Fr} R_{0} \sim R e^{\phi}$ with $\phi=0.5$. The higher-Reynolds-number cases fit the curve with $\phi=0.25$ below $R e=500$. Beyond $R e=500$, the flow becomes more turbulent and the quantity $Z_{m} / F r R_{0}$ approaches the scaling of Turner (1966) with the value of 2.46, as noted by Philippe et al. (2005).

The scaling $Z_{m} / R_{0} \sim F r \operatorname{Re}^{\phi}$ with $\phi=0.5$ is shown in figure 18 . The laminar and low-Reynolds-number transitional regions are fitted relatively well by the curve, but the higher-Reynolds-number points branch off the main curve. This suggests that fountain flow is not fully turbulent until above $R e=2000$. For this reason and the similarities with the images provided in Pantzlaff \& Lueptow (1999) and Turner (1966), we suggest that the flow is fully turbulent at $R e=2000$. This line has been tentatively marked on figure 2. Fully turbulent statistics for fountain flow were obtained from laboratory experiment by Cresswell \& Szczepura (1993) at $R e=2500$.

The good fit of the high-Froude-number laminar and transitional cases to the scaling of Philippe et al. (2005) with $\phi=0.5$ is not surprising. The authors covered 


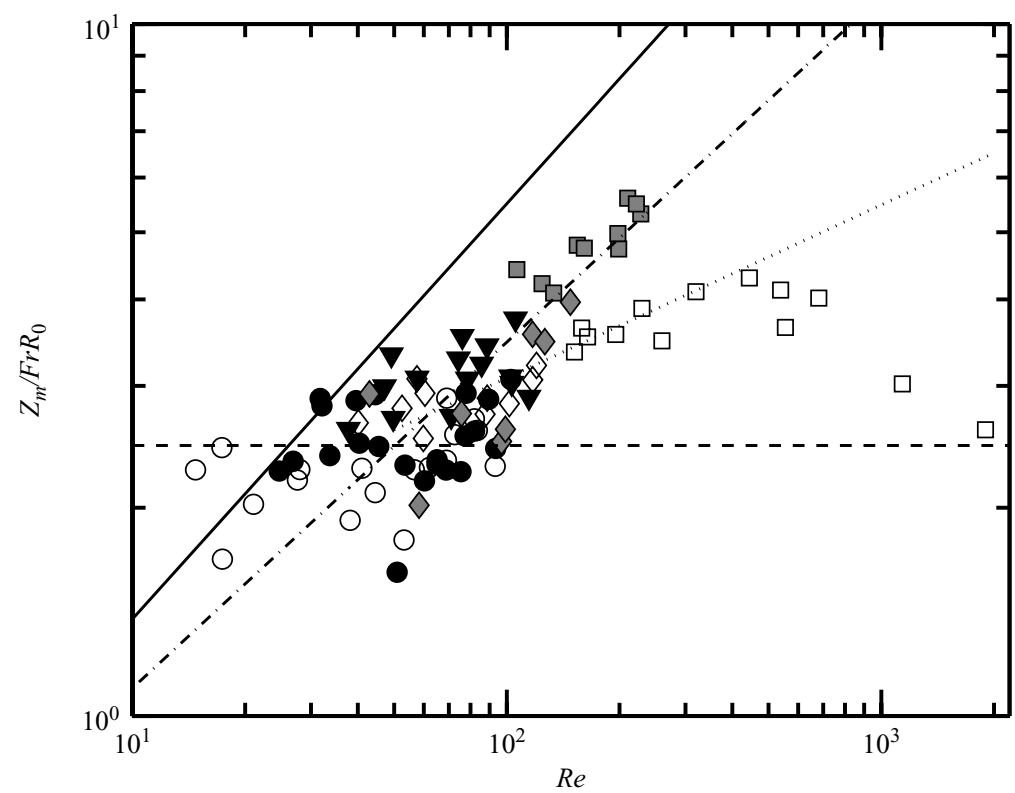

FiguRE 17. Constant for the linear Froude number scaling with Reynolds number, $C=Z_{m} / F r R_{0}$. Symbols defined in figure 2. Dashed line, 2.46 (Turner 1966); solid line, $0.347 R e^{0.6}$ (Philippe et al. 2005); dash dotted line, 0.347Re $e^{0.5}$ (Philippe et al. 2005); dotted line, $0.974 R e^{0.25}$ (Lin \& Armfield 2004).

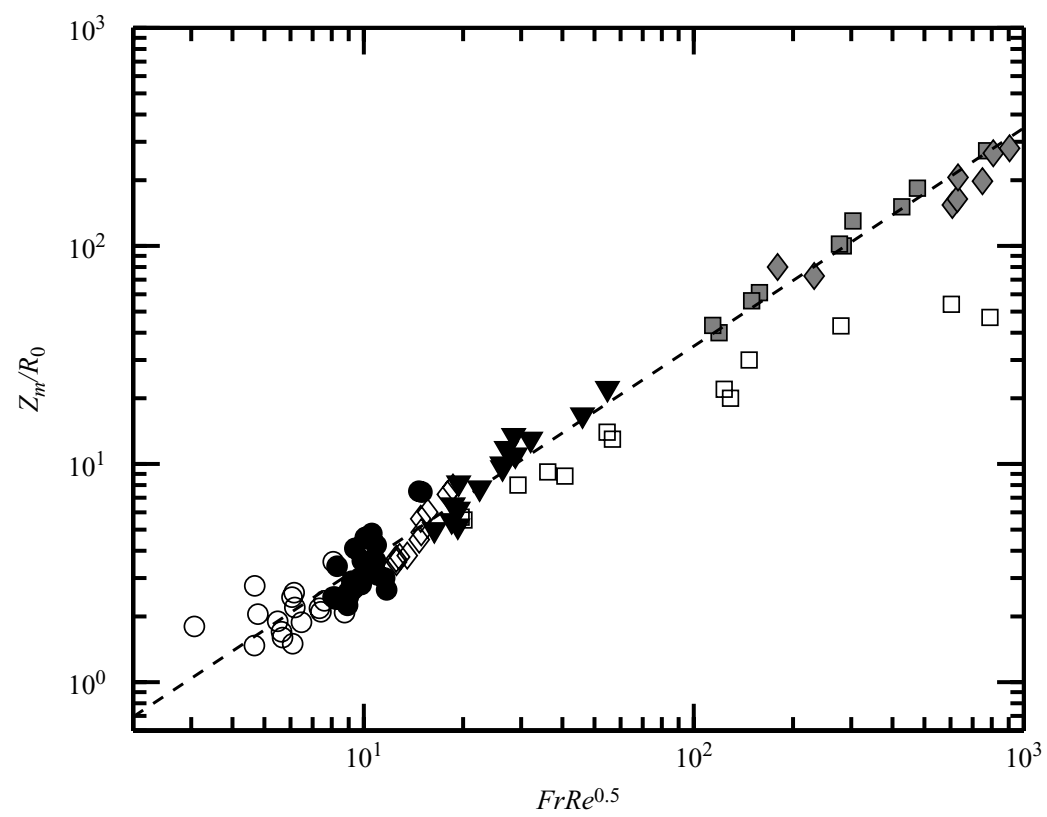

FIGURE 18. Mean maximum penetration height as function of Froude number and Reynolds number. Symbols defined in figure 2.

a similar experimental range and the flow observed here fits well with the analytical arguments the authors put forward, that is, that the maximum penetration height of the fountain is determined from a balance of the initial momentum and buoyancy 
together with viscous dissipation. Since only the fountain maximum penetration height is used here, not the average height, the instabilities and the fountain collapse have only a minor influence. Each time the fountain collapses, it rises again as a starting jet and reaches the maximum height with little interference of the previously collapsed fountain. Thus the important mechanisms are described well by the model. At higher Reynolds numbers, there is stronger mixing and entrainment in the rising column. Additionally, the descending flow interacts more with the rising flow so the analytical model does not apply, and thus the scaling breaks down.

In the low-Froude-number laminar and transitional cases, none of the scalings proposed to date appear to fit the data. The pulsing/bobbing flow is difficult to describe analytically; the manner of the descending flow and its interaction with the rising flow are complex.

\section{Conclusions}

The behaviour of low-Reynolds-number fountains has been investigated experimentally. Fountain flow has been found to be laminar for $R e<120$ independent of Froude number. Within the laminar region a range of previously unreported behaviour has been observed which varies with both Froude and Reynolds number. These behaviours have been located on a Reynolds and Froude number plane. The first unstable mode is a laminar flapping motion that can grow to a circling or multimodal flapping motion. In both laminar and transitional flow, for $F r \lesssim 10$, buoyancy forces dominate the flow and the fountain behaviour is characterized by the interaction between a succession of rising and falling fountain fronts, leading to bobbing and flapping behaviour in which there is little mixing with ambient fluid. The transition between steady behaviour, the initial flapping modes and more unsteady laminar bobbing flow can be approximately described by the function $\operatorname{Fr} \operatorname{Re}^{2 / 3}=C$.

High-Froude-number fountains $(F r \gtrsim 10)$ appear to be strongly dominated by a shear interaction between the rising and falling flow. For $R e<120$, this interaction appears to be responsible for a sinuous instability observed in the fountain column. At higher Reynolds number, this sinuous instability causes the fountain column to break down into turbulent jet-like flow, resulting in oscillatory behaviour with a period of $\sim 6 t \sigma / U_{0}$. The results in this study suggest that the flow becomes fully turbulent at $R e=2000$. At this point the shear-driven oscillation in fountain height is no longer observed.

The mean maximum penetration height of high-Froude-number laminar fountain flow is relatively well fitted by $Z_{m} / R_{0}=0.347 F r R e^{0.5}$ as proposed by Philippe et al. (2005). The relation does not apply as well to low-Froude-number laminar fountains. In the transitional region, the flow is more complicated and none of the previously proposed scalings apply well.

The authors wish to acknowledge the support of the Australian Research Council, and thank Atuski Komiya of Tohoku University for assistance with imaging.

\section{REFERENCES}

Abraham, G. 1967 Jets with negative buoyancy in homogeneous fluid. J. Hydraul Res. 5, 235-248. Baines, W. D., Turner, J. S. \& Campbell, I. H. 1990 Turbulent fountains in an open chamber. J. Fluid Mech. 212, 557-592.

Bloomfield, L. J. \& KerR, R. C. 2000 A theoretical model of a turbulent fountain. J. Fluid Mech. 424, 197-216. 
Campbell, I. H. \& Turner, J. S. 1989 Fountains in magma chambers. J. Petrol. 30, 885-923.

Clannet, C. 1998 On large-amplitude pulsating fountains. J. Fluid Mech. 366, 333-350.

Cresswell, R. W. \& Szczepura, R. T. 1993 Experimental investigation into a turbulent jet with negative buoyancy. Phys. Fluids A 5, 2865-2878.

Friedman, P. D. 2006 Oscillation height of a negatively buoyant jet. Trans. ASME J: J. Fluids Engng 128, $880-882$.

Friedman, P. D., Vadokoot, V. D., Meyer, W. J. \& Carey, S. 2007 Instability threshold of a negatively buoyant fountain. Exps. Fluids 42, 751-759.

Kaminski, E., Tait, S. \& Carazzo, G. 2005 Turbulent entrainment in jets with arbitrary buoyancy. J. Fluid Mech. 526, 361-376.

Kaye, N. B. \& Hunt, G. R. 2006 Weak fountains. J. F. Mech. 558, 319-328.

Lin, W. \& ARmField, S. W. $2000 a$ Direct simulation of weak axisymmetric fountains in a homogeneous fluid. J. Fluid Mech. 403, 67-88.

Lin, W. \& ARmfield, S. W. $2000 b$ Direct simulation of weak laminar plane fountains in a homogeneous fluid. Intl J. Heat Mass Transfer 43, 3013-3026.

Lin, W. \& ARmfield, S. W. 2000c Very weak axisymmetric fountains in a homogeneous fluid. Numer. Heat Transfer A 38, 377-396.

Lin, W. \& Armfield, S. W. 2003 The Reynolds and Prandtl number dependence of weak fountains. Comput. Mech. 31, 379-389.

Lin, W. \& ARMfield, S. W. 2004 Direct simulation of fountains with intermediate froude and reynolds numbers. ANZIAM J. 45, C66-C77.

Middleton, J. H. 1975 The asymptotic behavior of a starting plume. J. Fluid Mech. 72, 753-771.

Mizushina, T., Ogino, F., Takeuchi, H. \& IKawa, H. 1982 An experimental study of vertical turbulent jet with negative buoyancy. Wärme- und Stoffübertragung 16, 15-21.

PantzlafF, L. \& Lueptow, R. M. 1999 Transient positively and negatively buoyant turbulent round jets. Exps. Fluids 27, 117-125.

Philippe, P., Raufaste, C., Kurowski, P. \& Petitjeans, P. 2005 Penetration of a negatively buoyant jet in a miscible liquid. Phys. Fluids 17, 053601.

Srinarayana, N., McBain, G. D., Armfield, S. W. \& Lin, W. X. 2007 Height and stability of laminar plane fountains in a homogeneous fluid. Intl J. Heat Mass Transfer (submitted).

TuRnER, J. S. 1966 Jets and plumes with negative or reversing buoyancy. J. Fluid Mech. 26, 779-792.

ZhANG, H. \& BADDOUR, R. E. 1997 Maximum vertical penetration of plane turbulent negatively buoyant jets. J. Engng Mech. 123, 973-977.

ZhANG, H. \& BADDOUR, R. E. 1998 Maximum penetration of vertical round dense jets at small and large Froude numbers. J. Hydraul. Engng 124, 550-553. 